À André Roch Lecours (1936-2005)

Scientifique de renom, humaniste passionné et fondateur du Centre de recherche de l'Institut universitaire de gériatrie de Montréal

\section{Vieillissement normal et cognition}

Bernadette Ska, Yves Joanette
$>$ Le vieillissement normal est accompagné de modifications du fonctionnement cognitif. Les plus fréquentes touchent la mémoire, l'attention ou les habiletés visuospatiales. Des facteurs généraux et des facteurs spécifiques expliquent ces changements indicateurs du vieillissement cérébral. Ils ne sont cependant pas obligatoires, mais dépendent des réserves en ressources cognitives que les individus possèdent, et de la façon dont ils continuent à les entretenir et à les exploiter. Le cerveau est en effet un organe qui s'adapte si on le met dans de bonnes conditions. II est donc possible de retarder les effets du vieillissement et, dans certains cas, de les moduler. <

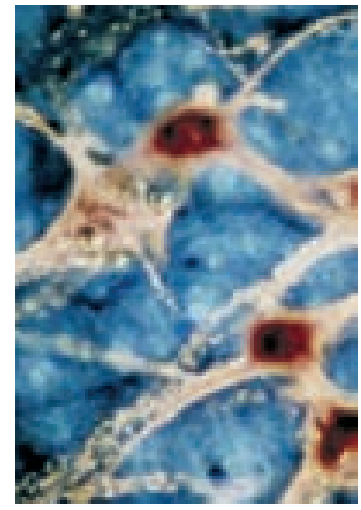

Centre de recherche, Institut universitaire de gériatrie de Montréal et Faculté de médecine, Université de Montréal, 4565, chemin de la Reine-Marie, Montréal (Québec), H3W IW5 Canada.

tinctes. Chacune de ces bernadette.ska@umontreal.ca fonctions est elle-même décomposable en plusieurs systèmes. Ainsi, la mémoire se décline en mémoire de travail ${ }^{\star 1}$, mémoire épisodique ${ }^{\star}$, mémoire sémantique ${ }^{*}$ et mémoire procédurale* ${ }^{*}$ De plus, un certain nombre de processus cognitifs (inhibition ${ }^{*}$, flexibilité mentale ${ }^{\star}$, vitesse de traitement ${ }^{\star}$ ) ne sont pas exclusifs à une fonction, mais contribuent à l'actualisation de plusieurs de ces fonctions et systèmes.

Le terme vieillissement revêt une connotation négative, alors que la réalité qu'il désigne est une notion toute relative. Pour paraphraser une maxime bien connue, on pourrait dire que chacun est plus vieux qu'hier, mais plus jeune que demain. Le vieillissement débute dès la conception d'un être, et il est irréversible dans son aspect chronologique et biologique. Cependant, la notion de vieillissement est très généralement associée à l'âge avancé et à ses manifestations négatives, en particulier dans le domaine de la cognition.

La cognition est considérée dans son sens large comme l'ensemble des capacités mentales permettant l'acquisition et le maintien de connaissances. Elle correspond à la faculté de traiter, de transformer et d'emmagasiner des informations pour les réutiliser. D'un point de vue théorique, la cognition comprend un ensemble de fonctions qui se distinguent soit par les caractéristiques des informations à traiter (langage, orientation spatiale), soit par le type de traitement à solliciter (mémoire, attention). Ces fonctions peuvent être perturbées de façon isolée, dans la mesure où elles dépendent de zones cérébrales disLe bon fonctionnement de la cognition dépend de l'intégrité de l'ensemble du cerveau. Ce dernier est un organe complexe, qui n'est ni stable ni inerte. Son organisation ne cesse d'évoluer et de s'adapter aux stimulations qu'il reçoit. Le fonctionnement du cerveau suit la croissance physique, et il ne peut atteindre un niveau optimal qu'à la fin de l'adolescence, lorsque la myélinisation des neurones est terminée. De nombreuses observations montrent que le cerveau continue à évoluer: d'ailleurs, il se réorganise au-delà de cette période, grâce à sa plasticité*. Une personne ne cesse jamais de traiter des informations; cependant la façon dont le cerveau traite les informations évolue avec l'âge.

Les spécialistes de la cognition s'entendent pour dire que le vieillissement s'accompagne d'une modification du fonctionnement cognitif, la plupart estimant qu'il évolue dans le sens d'un déclin [1]. Par exemple, les personnes âgées présentent des difficultés à manipuler et à traiter des informations visuelles ou spatiales, leur capacité à mémoriser diminue, elles ont de la difficulté

\footnotetext{
${ }^{1}$ Les termes ou groupes de termes suivis d'un astérisque sont définis dans le
} glossaire. 
à trouver le mot juste et parviennent plus difficilement à mener plusieurs tâches simultanément. Dans la suite du texte, nous allons résumer les connaissances actuelles concernant les altérations cognitives qui apparaissent avec le vieillissement normal. Nous aborderons les hypothèses actuelles qui expliquent ces modifications, ainsi que les facteurs qui accentuent ou ralentissent les effets du vieillissement normal sur le fonctionnement cognitif.

\section{Changements liés au vieillissement normal}

Les recherches sur le vieillissement normal montrent que les personnes âgées sont souvent considérées comme moins performantes que les personnes plus jeunes lorsqu'elles accomplissent des tâches qui sollicitent le fonctionnement de la mémoire, de l'attention, des capacités visuospatiales, du langage ou encore des fonctions exécutives* ${ }^{*}$ [1]. Ce déclin est relié aux changements que produit le vieillissement sur le système nerveux sur les plans neuroanatomique (diminution de la masse du cerveau), neurophysiologique (diminution du nombre et de la taille des neurones et perte de l'efficacité des contacts synaptiques) et neurochimique (diminution de la concentration de neurotransmetteurs, notamment, la dopamine) [2]. Les problèmes cognitifs observés chez les personnes âgées sont de divers ordres. D'un point de vue comportemental, les observations indiquent que les personnes âgées manifestent un ralentissement de leur vitesse de traitement de l'information, des difficultés à sélectionner les informations pertinentes, et à écarter celles qui sont moins pertinentes, des capacités diminuées à traiter deux types d'informations à la fois; ces opérations s'accompagnent d'une augmentation du nombre d'erreurs. Ces modifications cognitives ont des conséquences sur l'organisation, la qualité de la vie et la sécurité des personnes âgées et de leur entourage [3]. Malgré l'apparent consensus concernant les modifications de la cognition associées au vieillissement normal, de nombreuses questions restent ouvertes. Les principales concernent le caractère généralisé de ce déclin, sa direction et sa réversibilité [4]. En fait, l'évolution du fonctionnement cognitif est loin de constituer un processus simple.

\section{Différences inter- et intra-individuelles des effets du vieillissement}

Si une baisse d'efficience est admise pour l'ensemble des fonctions cognitives, les modifications observées ne revêtent pas un caractère linéaire. Certains domaines de la cognition sont altérés avant d'autres : par exemple, la mémoire est affectée avant les habiletés visuospatiales [5]. Plus spécifiquement, dans certains cas, certains systèmes relevant d'une même fonction suivent un déclin différent: par exemple, la capacité de la mémoire de travail ${ }^{\star}$ diminue avant d'autres systèmes de mémoire [6], et le traitement des mots, qui relève du système lexical du langage, est atteint avant le système phonologique, c'està-dire le traitement des sons de la langue [7]. En revanche, certains systèmes semblent mieux résister au vieillissement : ainsi, plusieurs aspects du langage se maintiennent avec l'âge, et il a même été montré, à de nombreuses reprises, que la mémoire sémantique (incluant la richesse du vocabulaire) ne cesse d'augmenter avec l'âge [8]. Des caractéristiques positives du vieillissement ont également été soulignées comme la sagesse, la maturité émotionnelle ou, encore, la capacité de développer des stratégies d'adaptation efficace [9].
Dans tous ces cas, l'accent est mis sur la différence de façon d'agir entre les personnes âgées et les personnes plus jeunes. Les observations précédentes proviennent de recherches ayant comparé la façon d'agir de personnes âgées avec celles de personnes plus jeunes. D'autres recherches, qui ont comparé les personnes âgées entre elles, arrivent à la conclusion que l'évolution de leurs habiletés cognitives ne suit pas toujours un pattern temporel ou «patron» similaire. Certaines personnes gardent un niveau élevé d'efficacité identique très longtemps, d'autres présentent des modifications plus rapidement [10]. De plus, lorsque des altérations apparaissent, elles ne se manifestent pas toujours selon le même profil [11] : certaines personnes présentent ainsi des difficultés dans l'exécution de tâches verbales, tandis que d'autres éprouvent plus de difficultés dans l'accomplissement de tâches visuospatiales.

\section{Faut-il considérer ces changements comme l'expression de déficits ou comme la mise en place de stratégies adaptatives?}

La réponse à cette question n'est ni simple, ni claire. Une première tentative de réponse consiste à rechercher un facteur commun sous-jacent à l'émergence des différents déficits. Les processus cognitifs de base, dont on considère l'altération responsable de la baisse d'efficience cognitive générale, sont la vitesse de traitement ${ }^{\star}$, la mémoire de travail ${ }^{\star}$ et la résistance à l'interférence ${ }^{\star}$ ou capacité d'inhibition ${ }^{\star}$ [12]. De nombreuses études corrélationnelles ont mis en évidence la primauté de l'un ou l'autre de ces trois facteurs, les résultats étant liés aux types de tests ou de tâches utilisés pour évaluer le fonctionnement cognitif des personnes âgées. Un quatrième facteur, plus général, a également été proposé : il s'agit des réserves de ressources cognitives $^{\star}$ dont dispose un individu pour agir. Baltes [13] distingue deux types de ressources: les ressources de base et les réserves développementales. Le premier type définit la capacité des ressources sur lesquelles un individu peut compter, tandis que le second exprime le potentiel qui lui permettrait d'augmenter ses ressources de base par apprentissage ou entraînement. Ce concept a son corollaire cérébral : la plasticité*.

Dans cette perspective, la manifestation des effets du vieillissement dépendra, en partie du moins, de caractéristiques innées ou acquises lors du développement, caractéristiques qui peuvent moduler les réserves cognitives. II s'agit, par exemple, des styles cognitifs (analytique versus global ${ }^{\star}$ ), du genre et du niveau d'éducation, de la personnalité, de l'état de santé, du type de connaissance ou d'expérience (déterminé généralement par les études ou la profession) et du style de vie. Par ailleurs, bon nombre de travaux montrent que l'isolement social, la privation de sommeil, la dépression, l'anxiété et une faible estime de soi sont des facteurs qui contribuent à l'apparition des altérations cognitives ou à leur exacerbation [14-18]. 
Tous ces facteurs façonnent différemment les comportements des individus. Ainsi, des études montrent que les femmes âgées sont plus susceptibles d'avoir des problèmes visuospatiaux que les hommes âgés [16]. Inversement, les capacités verbales des femmes sont supérieures à celles des hommes. Par ailleurs, le niveau d'éducation [19] et le style de vie [20] sont considérés comme de bons indicateurs des ressources cognitives. Ces facteurs contribuent donc à moduler les ressources disponibles chez les individus.

Les études réalisées en neuro-imagerie fonctionnelle ont contribué à une meilleure connaissance du fonctionnement cérébral, et fournissent des indications concernant les modifications observées chez les personnes âgées. Lors de la réalisation de deux tâches théoriquement différentes si l'on en juge par les ressources cognitives qu'elles exigent, la neuro-imagerie fonctionnelle suggère que le recours aux réserves de ressources nécessaires à la tâche plus exigeante se marque par l'activation de zones cérébrales supplémentaires. Ainsi, dans des tâches attentionnelles, Weissman et Banich [21] ont observé que lorsque la demande est minimale, un seul hémisphère cérébral est activé. Lorsque la tâche se complexifie, la demande en ressources augmente et les deux hémisphères s'activent. Le cerveau s'adapte donc, et appelle la contribution de plusieurs zones si des ressources supplémentaires sont nécessaires. Des observations semblables ont été faites en comparant la réalisation d'une même tâche par des personnes jeunes et âgées: elles ont contribué à la proposition du modèle Harold (hemispheric asymetry reduction in older adults) par Cabeza $[22,23]$. Selon ce modèle, pour assurer le maintien de leurs habiletés cognitives, les personnes âgées feraient appel à un réseau neuronal distribué sur les deux hémisphères cérébraux pour réaliser certaines tâches, tandis que les personnes plus jeunes n'utilisent généralement qu'un seul hémisphère. Cabeza et ses collaborateurs proposent donc que le vieillissement réussi s'accompagne d'une dédifférenciation* de la latéralisation cérébrale. Cette dédifférenciation n'est qu'apparente si l'on fait référence à l'hypothèse, proposée par Weissman et Banich, de l'activation d'un deuxième hémisphère pour augmenter les ressources disponibles. En effet, si les personnes âgées comptent moins de ressources pour la réalisation d'une tâche, elles peuvent faire appel à des ressources supplémentaires en faisant intervenir le deuxième hémisphère. Cette interprétation va dans le sens d'une adaptation des personnes âgées par l'utilisation de stratégies compensatoires, indiquant ainsi que le cerveau maintient une certaine plasticité*.

\section{Conditions pour un vieillissement cognitif optimal}

De nombreux travaux de recherches ont montré que les personnes âgées les plus en forme sont celles qui restent actives sur le plan cognitif [20]. On peut affirmer que les activités favorables au vieillissement réussi sont celles qui font appel aux fonctions dites exécutives ${ }^{\star}$ : planification, attention, sélection, inhibition * et coordination $[24,25]$. Parmi ces activités, on peut citer des activités manuelles, telles que le jardinage, la menuiserie, le tricot ou la couture, ou des activités plus intellectuelles, telles que le bridge, les mots-croisés, l'écriture et la lecture. De plus, chez certaines personnes, un entraînement cognitif ciblé* peut augmenter l'efficacité de leurs activités, indiquant ainsi que la diminution n'est pas irréversible [26]. Enfin, plusieurs facteurs concourent au maintien du fonctionnement cognitif optimal, notamment l'activité physique [27], la qualité du sommeil [18], l'absence de maladie [28], le réseau et l'engagement social [14, 17].

\section{Conclusions}

Dans une perspective de prévention, la recherche qui s'applique aux conditions favorables à un vieillissement réussi est prometteuse, et permet de plus en plus d'entrevoir l'élaboration de modalités favorables à un vieillissement réussi ou optimal [29]. Encore sera-t-il nécessaire d'intégrer l'ensemble des conditions favorisant un «bien vieillir», notamment la nutrition et l'activité physique. En définitive, les résultats des travaux montrent que la réussite de la vieillesse tient à la combinaison de trois ensembles de conditions: l'absence de maladie menant à une perte d'autonomie, le maintien des activités intellectuelles et physiques ainsi que l'engagement social et le bien-être subjectif. La convergence de ces conditions dépend autant de facteurs personnels que de facteurs sociaux et environnementaux. $\diamond$

\section{SUMMARY}

Normal aging and cognition

It is now well documented that normal aging modifies the cognitive functioning and most observations suggest that cognition evolves in the direction of deterioration. The more frequently impaired functions are memory, attention and visual-spatial abilities. On the other hand, some abilities seem to increase, such as vocabulary. Considering the aging effect on cognition, questions remain regarding directionality, universality and reversibility. A great variability in aged related impacts is observed among subjects and among cognitive domains. Some individuals evolved more rapidly than others. Some cognitive functions are more affected by aging than others. General and specific factors are hypothesized to explain the aged related cognitive decline. Among them, educational level, health, cognitive style, life style, personality, are likely to modulate the aged related cognitive evolution by influencing attentional resources and cerebral plasticity. Cognitive resources are essential to develop adaptative strategies. During the life span, resources are activated and increased by learning and training. Considering the role of cognitive resources, successful aging is dependent on several conditions: absence of disease leading to a loss of autonomy, maintenance of cognitive and physical activities, and active and social engaged lifestyle. $\diamond$

\section{REMERCIEMENTS}

Les auteurs tiennent à remercier les évaluateurs anonymes pour leurs judicieux conseils.

\section{RÉFÉRENCES}

1. Van der Linden M, Hupet M. Le vieillissement cognitif. Paris : PUF, 1994.

2. Raz N. Aging and the brain and its impact on cognitive performance : integration of structural and functional findings. In : Craik FIM, Salthouse TA, eds. The handbook of aging and cognition. New Jersey: Lawrence Erlbaum Associates, 2000 : 1-90. 


\section{GLOSSAIRE}

Dédifférentiation cérébrale : hypothèse suggérée par Cabeza [22, 23] à la suite des observations selon lesquelles les personnes âgées maintiennent un niveau de performance équivalente à celui des plus jeunes en faisant appel à des zones cérébrales distribuées sur les deux hémisphères, alors que la même tâche est latéralisée (dépend d'un seul hémisphère) chez les personnes plus jeunes. Il y aurait moins de différences entre les deux hémisphères chez les personnes âgées, en comparaison des plus jeunes, quant à l'activation des zones cérébrales lors de la réalisation d'une tâche.

Entraînement ciblé : programmes visant l'entraînement d'une fonction en particulier (par exemple, une meilleure utilisation de la mémoire ou une augmentation des capacités attentionnelles).

Flexibilité mentale : capacité à détourner rapidement son attention d'une situation à une autre et à répondre de façon adaptée à la nouvelle situation.

Fonctions exécutives : capacité à adopter et à poursuivre un comportement en vue d'atteindre un but précis.

Interférence: situation où deux ou plusieurs informations sont en compétition dans la réalisation d'une tâche. On parle de résistance à l'interférence si la personne est capable d'ignorer les informations qui seraient non compatibles avec la réalisation de la tâche qu'elle doit exécuter.

Inhibition : capacité à réprimer une réaction ou une réponse en présence d'un stimulus qui normalement devrait l'activer.

Mémoire de travail : capacité limitée à maintenir et à traiter plusieurs informations en même temps durant un court laps de temps.

Mémoire épisodique : mémoire des faits vécus, mémoire biographique, mémoire des informations dépendantes d'un contexte précis en termes de lieu, de temps et de circonstances.

Mémoire procédurale: mémoire des gestes appris (à distinguer des réflexes) difficilement verbalisables.

Mémoire sémantique: mémoire des connaissances générales sur le monde, incluant les faits, les concepts et le vocabulaire.

Plasticité cérébrale : capacité du cerveau à se réorganiser, en termes de connexions neuronales, pour répondre à de nouvelles demandes. La réorganisation peut se faire à la suite d'une lésion, mais aussi à la suite d'un apprentissage ou d'un entraînement.

Ressources cognitives : capacité d'une personne à réaliser simultanément plusieurs tâches qui exigent de gérer plusieurs informations ; la capacité se définit en termes de quantité (combien) et de qualité (comment). Les ressources cognitives incluent les capacités attentionnelles.

Style cognitif analytique ou global : le style cognitif caractérise la manière privilégiée dont une personne traite, enregistre ou organise l'information. Le style analytique correspond à une approche séquentielle qui progresse par découpage, recherche des détails et des principes d'organisation; le style global préfère une approche holistique, compréhensive, simultanée et imagée.

Vitesse de traitement: donnée comportementale de base estimée en temps de réaction (un stimulus simple) ou de réponse (situation à choix de réponse fermé tel que oui ou non).
5. Albert M, Duffy FH, Naeser M. Nonlinear changes in cognition with age and their neuropsychologic correlates. Can J Psychol 1987 ; 41 : 141-57.

6. Craik FIM. A functional account of age differences in memory. In : Klix F, Hagendorf $\mathrm{H}$, eds. Human memory and cognitive capabilities : mechanism and performance, part A. Amsterdam : Elsevier, 1986 : 409-22.

7. Balota DA, Duchek JM. Age-related differences in lexical access, spreading activation, and simple pronunciation. Psychology Aging 1988; $3: 84-93$.

8. Verhaeghen P. Aging and vocabulary scores : a meta-analysis. Psychology Aging 2003; 18 : 332-9.

9. Light LL. Memory changes in adulthood. In : Qualls SH, Abeles N, eds. Psychology and the aging revolution: how we adapt to longer life. Washington, DC, US : American Psychological Association 2000, IX : 73-97.

10. Ylikoski R, Ylikoski A, Keskivaara $P$, et al. Heterogeneity of cognitive profiles in aging: successful aging, normal aging, and individuals at risk for cognitive decline. Eur J Neurol 1999; 6: 645-52.

11. Valdois $S$, Joanette $Y$, Poissant $A$, et al. Heterogeneity in the cognitive profile of normal elderly. J Clin Exp Neuropsychol 1990; 12 : 587-96.

12. Van der Linden $M$, Hupet $M$, Feyereisen $P$, et al. Cognitive mediators of age-related differences in language comprehension and verbal memory performance. Aging Neuropsychology Cognition 1999; 6 : 32-55.

13. Baltes PB. On the incomplete architecture oh human ontogeny : selection, optimization, and compensation as foundation of developmental theory. Am Psychologist 1997 ; 52 : 366-80.

14. Seeman TE, Lusignolo TM, et al. Social relationships, social support, and patterns of cognitive aging in healthy, high-functioning older adults: MacArthur studies of successful aging. Health Psychology $2001 ; 20: 243-55$.

15. Rowe JW, Kahn RL. Successful aging. Gerontologist 1997 ; 37 : 433-40.

16. Parsons TD, Rizzo AR, van der Zaag C, et al. Gender differences and cognition among older adults. Aging0 Neuropsychology Cognition 2005; 12 : 78-88.

17. Bosworth HB, Schaie KW. The relationship of social environment, social networks, and health outcomes in the Seattle longitudinal study: two analytical approaches. J Gerontol Series B Psychological Sciences and Social Science 1997 ; 52 : 197-205.

18. Cohen-Zion M, Stepnowsky CM, Shochat T, et al. Changes in cognitive function associated with sleep disordered breathing in older. J Am Geriatr Soc 2001 ; 49: $1622-7$.

19. Herrera-Guzman I, Pena-Casanova J, Lara JP, et al. Influence of age, sex, and education on the Visual Object and Space Perception Battery (VOSP) in a healthy normal elderly population. Clin Neuropsychologist 2004 ; 18 : 385-94.

20. Newson RS, Kemps \&B. General lifestyle activities as a predictor of current cognition and cognitive change in older adults : a cross-sectional and longitudinal examination. J Gerontol Series B Psychological Sciences and Social Science 2005 ; 60B : 113-20.

21. Weissman DH, Banich MT. The cerebral hemispheres cooperate to perform complex but not simple tasks. Neuropsychology $2000 ; 14: 41-59$.

22. Cabeza R. Hemispheric asymmetry reduction in older adults : the HAROLD model. Psychology Aging $2002 ; 17: 85-100$.

23. Cabeza R, Daselaar SM, Dolcos F, et al. Task-independent and task-specific age effects on brain activity during working memory, visual attention and episodic retrieval. Cerebral Cortex 2004 ; 14 : 364-75.

24. Le Carret N, Lafont S, Mayo W, Fabrigoule C. The effect of education on cognitive performances and its implication for the constitution of the cognitive reserve. Dev Neuropsychol $2003 ; 23$ : 317-37.

25. Fabrigoule C. Do leisure activities protect against Alzheimer's disease? Lancet Neurol $2002 ; 1: 11$.

26. Kramer AF, Willis SL. Enhancing the cognitive vitality of older adults. Curr Dir Psychol Sci $2002 ; 11: 173-7$.

27. Colcombe $S$, Kramer AF. Fitness effects on the cognitive function of older adults : a meta-analytic study. Psychol Sci $2003 ; 14$ : 125-30.

28. McCurry $S M$, Gibbons $L E$, Bond $G E$, et al. Older adults and functional decline : a cross-cultural comparison. Int Psychogeriatr $2002 ; 14$ : 161-79.

29. Baltes PB, Baltes MM. Psychological perspectives on successful aging: The model of selective optimization with compensation. In: Baltes PB, Baltes MM eds. Successful aging : perspectives from the behavioural sciences. Cambridge : Cambridge University Press, $1990: 1-34$.

3. Studer M. Cognitive rehabilitation in the frail elderly patient never too old to learn? Topics Geriatr Rehabil $2004 ; 20: 21-33$.

4. Dixon RA. Themes in the aging of intelligence: Robust decline with intriguing possibilities. In : Sternberg, Robert J Lautrey J, eds. Models of intelligence : international perspectives. Washington, DC, US : American Psychological Association, 2003, XVI : 151-67.

\section{TIRÉS À PART}

B. Ska 